\title{
Research on Data Synchronization Technology of Embedded Mobile Database
}

\author{
Changai Chen ${ }^{*}$ and Xiaohui Wang
}

\author{
School of Information Technology, Henan University of Traditional Chinese Medicine, Zhengzhou, Henan 450000, \\ China
}

\begin{abstract}
This paper stresses on the Management algorithm of Client Cache in Embedded Mobile Database, and mainly extends on three aspects: the Replacement Algorithm, Prefetching Algorithm, and non-consistency of data. The Cache Management of mobile device is based on the mobile database running on multi-disk broadcast environment, which is characterized as not requiring upload channel. In this case, the client can continuously receive expected data stream without sending requests to the server. On the other hand, according to client access requests, the server can attempt to broadcast the data item in sequence so as to make different data items broadcast at different frequencies.
\end{abstract}

Keywords: Data Synchronization, Embedded Mobile Database, Information System.

\section{INTRODUCTION}

In future, Internet of things, Big Data and cloud computing will become three major technologies of IT (Information Technology) industry, while data synchronization technology in embedded mobile database is a core method in these technologies. Embedded mobile computing technology has become a research focus in the fields of database and wireless network, and has been paid much attention. Researchers and industries pay greater attention to the study of the embedded mobile database. Specially, logistics industries have encountered rapid development under the application of embedded mobile database technology. However, there are a lot of shortcomings in the design of logistics management systems used at present, which are shown in data acquisition, data transmission, data organization and data synchronization in the mobile databases. In the CPU, memory, storage past achievements and related applications have paved the road for the next generation, in local data. These data can be [1] (for example, server and desktop computers, the application of embedded devices, in any place (local) on PDA, remote access and closing network connection) price decline and 64 bit addressing. Obviously, it is unusual for $8,16 \mathrm{~GB}$ of internal memory to configure the server, storage and $\mathrm{PC}$ desktop using flash technology. Due to reasonable price of the flash drive, bietet.32 GB flash drive, the city's IU C, pgfla by potential computer, $\mathrm{U}$ disk not only eliminates the time consumption, but also enhances the power of traditional search which is difficult for the hard disk to accommodate. So, it naturally closes the portable lattice te.das, composite material and processes mobile applications and other data in the application (2)]. Components can be different at different levels as different services range on different platforms (e.g., servers, PCs, laptops).

*Address correspondence to this author at the School of Information Technology, Henan University of Traditional Chinese Medicine, Zhengzhou, Henan 450000, China; E-mail: 13503453662@163.com
As a kind of new rising technology, Mobile Embedded Database is an important branch of traditional distributed database $[2,3]$. Due to special running environment, technology used in distributed database system cannot proceed in Embedded Mobile Database. Therefore, further research on Embedded Mobile Database technology has become a hotspot in this field.

\section{SYSTEM FRAME}

Embedded Mobile Database System is a database management system which supports mobile computing or some specific computing mode. Database system is integrated with operating system and application and operates in various intelligent embedded equipments or mobile equipment [4]. Embedded Mobile Database includes some key technologies such as replication and caching of data, data broadcasting, query and optimization of position, process of mobile transaction.

Modern logistics is a kind of service industry, in which computer management and communication technologies are crucial techniques. With respect to the current situation of logistics industries, data synchronization technique is studied in embedded mobile database design [5]. The focus is on the data acquisition of the embedded mobile terminal, data transmission and synchronization between embedded mobile terminals and servers, establishment and maintenance of server databases, so as to solve some problems such as data acquisition and data synchronization, complex equipment operation, data overload, data error and data loss in data transmission. The disposal method here is to achieve information anytime and anywhere by embedded portable devices, to simplify operation of mobile terminals with Android APP (Fig. 1), that is to diminish errors and to enhance the safety and reliability of data by techniques of breakpoint resume, Cache and synchronization.

Embedded Mobile Database, which runs on motive embedded devices, is an essential part of the whole mobile da- 


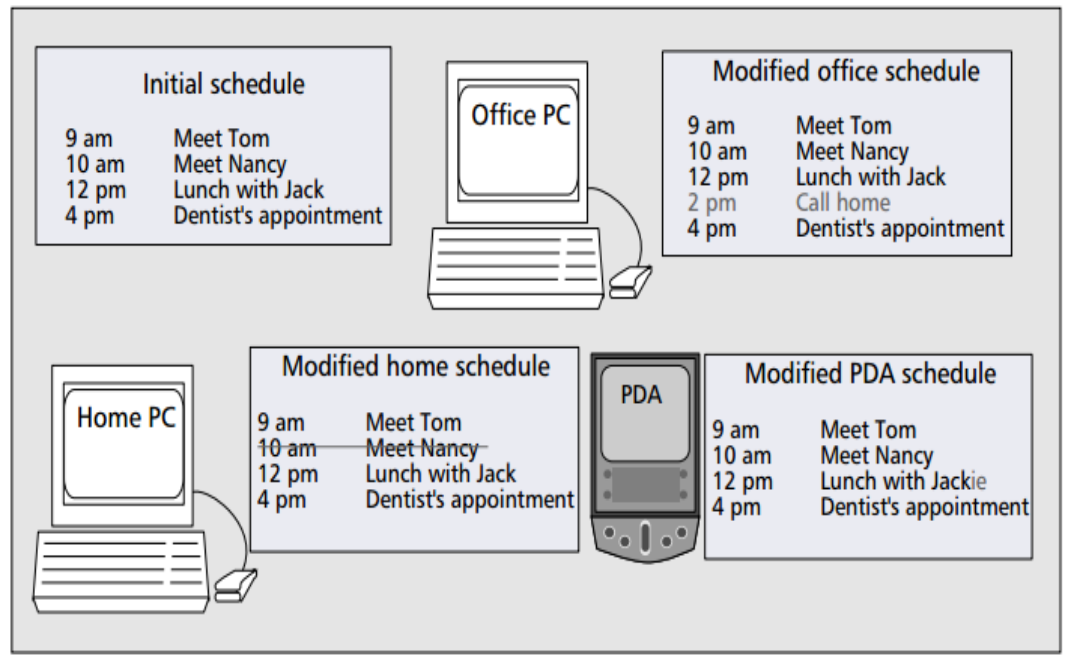

Fig. (1). An appointment schedule modified on different devices: a home machine, an office machine, and a PDA.

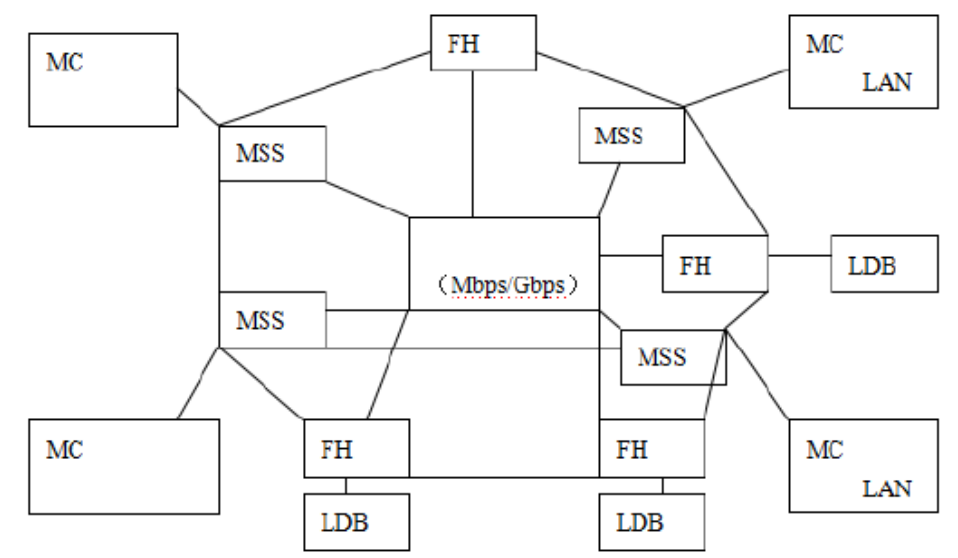

Fig. (2). The system architecture of embedded mobile database.

tabase system. Instead of being analyzed and researched in isolation the technology involved should be extended and deepened within the whole system. This paper starts from the model of Mobile Database System, analyzes the function of Embedded Mobile Database in the entire system, and explores thoroughly and studies deeply the key techniques in this field according to the motive characteristics, such as the motion of client, the frequent break and connection between client and network, the variety of network condition, the non-symmetry of network communication and so on (Fig. 2).

The paper is organized as follows. Firstly, the Replacement Algorithm of Cache Management was studied by analyzing two sutra Algorithms: LRU and 2Q. The suitable Cache Replacement Algorithm of multi-disk broadcast---PIX Algorithm is proved by using mathematical method. Through these analyses and investigations, an ideal algorithm-2QIX has been provided, which can run stably in multi-disk broadcast environment (Fig. 3). This algorithm has integrated the advantages of $2 \mathrm{Q}$ and PIX algorithm. It considers not only the issue of the associated access of data item, but also of the broadcast frequency gene. It can estimate the future access probability of data item via the history access records of data item, and, by experiments, it can turn out to have better effects.
Secondly, the Perfecting Algorithm is discussed. At the beginning, a reasonable Algorithm-PT algorithm is analyzed. Using Perfecting Algorithm, the client can continuously receive expected data items in broadcast environment. Therefore, Perfecting Algorithm works quite well in such an environment. However, PT algorithm has its restrictions in practice. In this case, a feasible Perfecting algorithm-EPT algorithm is provided. This algorithm makes PT algorithm possible by estimating the future access probabilities via the history access records of data items. But it demands the mobile device having better computing ability, which can complete the comparison of pt value at every broadcast unit interval.

Thirdly, non-consistency of data for Cache Management is discussed (Fig. 4). Considering the necessity and inevitability of Data non-consistency in application environment, two key solutions to the problem are provided: Invalidation Setting and Propagation Updating Value. Since these two methods have their own features, it can be a pretty good solution if they are combined together according to their different statuses.

Finally, the executable model of Mobile Database system is built, testifying all algorithms discussed throughout the paper and their performances are validated through trials 


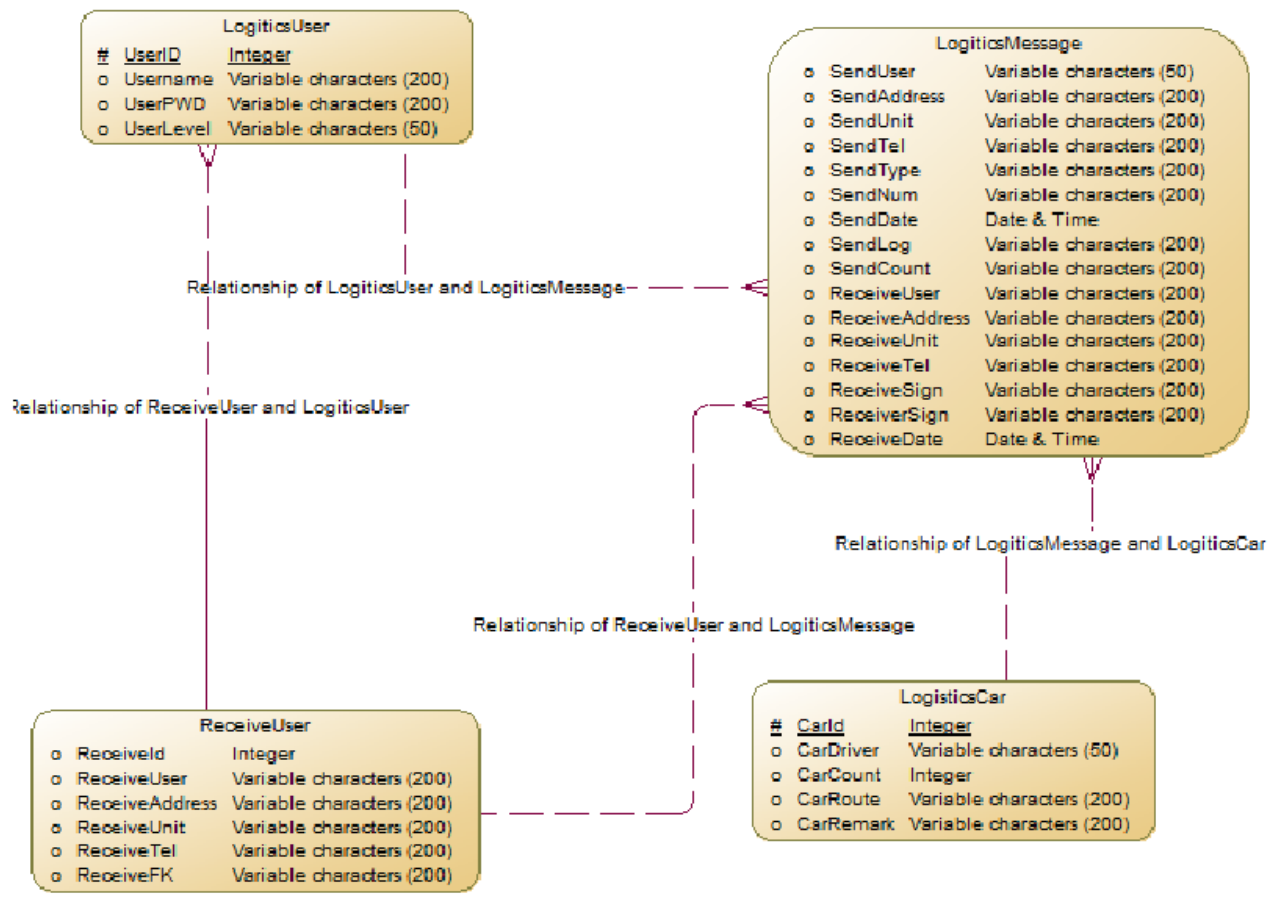

Fig. (3). The data model of CDM concept.

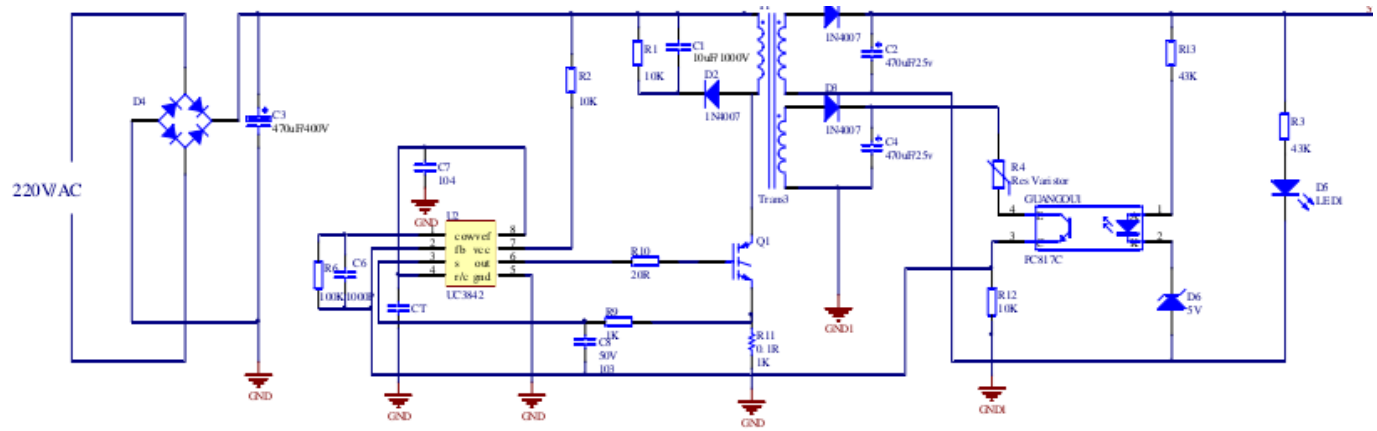

Fig. (4). Power supply design of AC-DC.

(Fig. 5). The conclusion of this paper can have certain contribution in the application of Embedded Mobile Database.

The logistics database synchronization system proposed is composed of three modules that is, hardware of the embedded mobile terminals, transmission and synchronization of data processing of server database. The core component of the embedded mobile terminals consists of a CPU of CortexA8, GPS, network cards, keys and display screens etc, in order to evaluate order form information acquisition and supervisory control online of logistics systems. Data transmission and synchronization involve $3 \mathrm{G}$ technologies as premise of data transmission and synchronization, Internet work as supporter, cache and FIFO algorithm as techniques, transfer data information of embedded mobile terminals to former server. Server database incorporates charge data maintenance and processing, realizing the instantaneity of data, and provides server for on-line inquiry. The three parts supplement each other, collectively realizing data synchronization of whole logistics database, so as to meet the needs of real-time business management of logistics industry, gain integrated management model of data flow and information flow, supply an informative platform of logistics management and to promote the management level of logistics industry.

\section{EMBEDDED}

In the crowded simple computer technology tools are needed to be sought for storage and operation of data and application. These declarations, however are not hard and have been developed to meet the needs of a particular user. Usually these applications render vertical development in key industries, such as medical, financial and other aspects, but these enterprises do not require a database expert in the database installation for configuration and management of their time. Focus on embedded applications, application and database synchronization is very important, usually from the perspective of application developers. Database is a typical single application database which does not involve sharing (or coordinates) of other databases (this is service of database application, for example, equipment). "Korolev", the author, are its author computers, desktop computers, servers, 


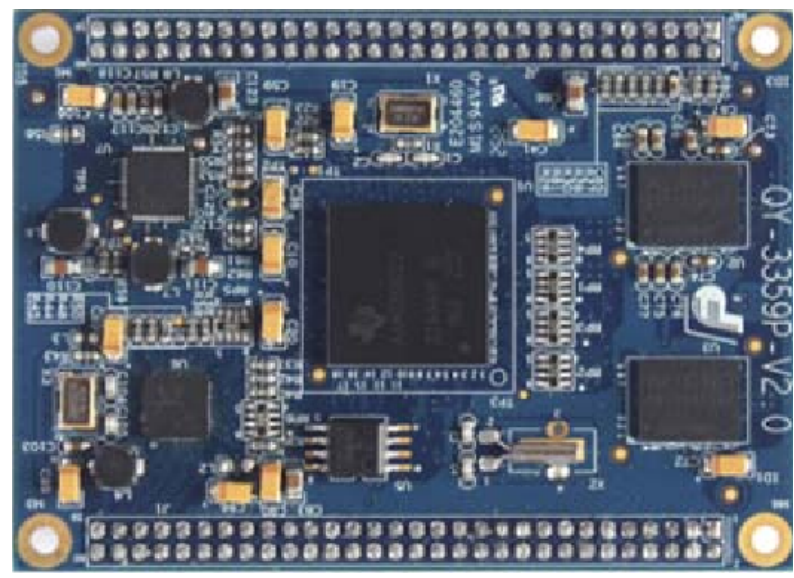

Fig. (5). The main control board of A8 minimum system.

server using the ideal choice. In the use of components and applications as the only life, different applications have different needs, some from the database access and other common problems, the need to support, and also some people may with the backstage database synchronization, embedded application function component is the decisive condition.

$$
\begin{aligned}
& \mathrm{S}_{\mathrm{k}}=\sum_{i \in D} \mathrm{P}_{\mathrm{i}} \cdot l_{\mathrm{i}}=\sum_{i \in C_{\mathbf{k}}} \mathrm{P}_{\mathrm{i}} \cdot l_{\mathrm{i}}+\sum_{i \in C_{\mathrm{k}}} \mathrm{P}_{\mathrm{i}} \cdot l_{\mathrm{i}}=\sum_{i \in C_{\mathbf{k}}} \mathrm{P}_{\mathrm{i}} \cdot 0+\sum_{i \in C_{\mathbf{k}}} \mathrm{P}_{\mathrm{i}} \cdot l_{\mathrm{i}}=\sum_{i \in C_{\mathbf{k}}} \mathrm{P}_{\mathrm{i}} \cdot l_{\mathrm{i}} \\
& \mathrm{S}_{\omega+1}=\sum_{l \in C_{\mathrm{k}}} \mathrm{P}_{\mathrm{i}} \cdot l_{\mathrm{i}} \\
&=\mathrm{S}_{\omega}-\mathrm{P}_{a_{\omega+1}} \cdot l_{a_{\omega+1}}+\mathrm{P}_{v} \cdot l_{v} \\
&=\mathrm{S}_{\omega}+\mathrm{P}_{v} \cdot 1_{v}
\end{aligned}
$$

The rapid development of the application of vertical (Embedded Systems) and traditional database provider does not meet the requirements of component manufacturers, whether differences in management application or data and developers, every day, has technology, the basic document, store and retrieve data. Or, in particular of the management team, is the need to fill in some of the products of embedded technology. Application developers want from the database, database providers.

\subsection{Key Codes}

final String createLoginsticsUserTable="create table LogisticsUser"+ text

(Userld integer primary key autoincrement, UserName

text,UserLevel text)";

final String createReceiverUserTable $=$ "create

table ReceiverUser(ReceiverUser text,"+

"ReceiveAddress text,ReceiveUnit text,"+

final String createLoginsticsMessageTable ="create table LogisticsMessage(SendUserVarchar

text,SendAddress text,SendUnit text,Send pe text,SendNum text,"+

"SendDateDate text,SendLog text,"+

"SendCount

text,ReceiveUser

\author{
text,ReceiveAddress \\ text,ReceiveUnit \\ text,ReceiveTel text,ReceiveSign text,ReceiverSign \\ text,ReceiveDate time)" \\ "ReceiveTel text,ReceiveFK text)"; \\ *@param context \\ *@param name \\ *@param version \\ $* /$
}

public MyDatabaseHelper(Context context, String name, int version) \{

super(context, name, null, version);

\}

(a) Override

public void onCreate(SQLiteDatabase $\mathrm{db}$ )

db.execSQL(createLoginsticsUserTable);

db.execSQL(createReceiverUserTable);

db.execSQL(createLoginsticsMessageTable);

(a) Override

public void onUpgrade(SQLiteDatabase $\mathrm{db}$, int oldVersion, int newVersion) \{

System.out.println("--------onUpdate Called----- ” +old Version

+” -> + +new Version);

\subsection{Method}

Analysis regarding conflict problem of replication technique has brought out Two-Tier Replication Scheme and Synchronization Replication Scheme in light of the conflict problem:

1. Two-Tier Replication Scheme adopts bidirectional and synchronous replication method. Embedded Mobile Database detects and solves conflict of renewed data which database server downloads to synchronous server. And Embedded Mobile Database uploads renewed data to synchronous server. Synchronous server detects conflict of renewed data. Synchronization Replication Scheme adopts upload transac- 
tion arithmetic based on Relate Transaction Scheme, upload transaction arithmetic based on Time Limit Scheme and Relate Transaction Scheme- Time Limit Scheme replication arithmetic based on two conflicts solve scheme. The timing complexity of upload arithmetic is $\mathrm{O}$ (n) (Fig. 6).

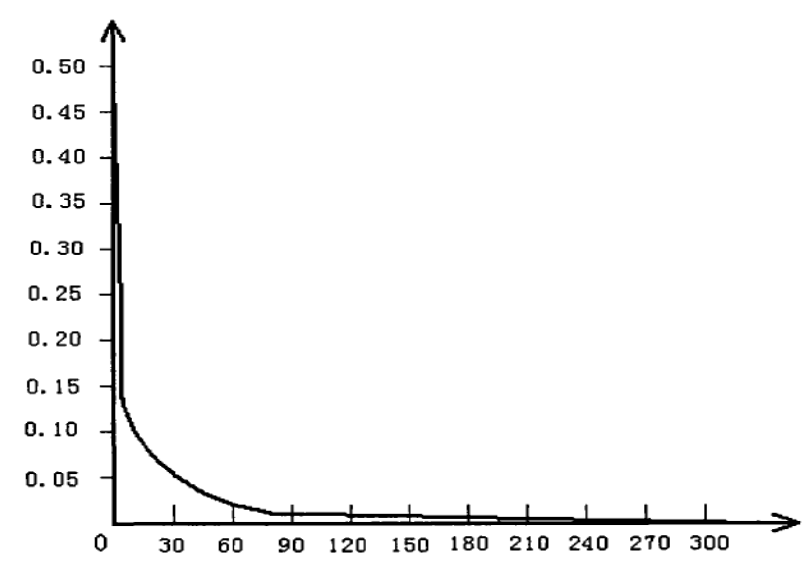

Fig. (6). Run results.

2. When validating the experiment, the result of TwoTier Replication Scheme in Embedded Mobile Database can detect and solve conflict before submitting tentative transaction. It can avoid transfer of cancelled transaction to database server. Moreover, it can reduce the quantity of data transmission and enhance autonomy of Embedded Mobile Database. The result of Synchronization Replication Scheme is the quick time for detecting the conflict. It can enhance efficiency of synchronization as well.

The two solutions aiming at confliction can achieve final consistency of the whole Embedded Mobile Database System in application. And these can also enhance user testing of Embedded Mobile Database application.

Essentially, the wireless network is far from fixed network security, because it is difficult to listen and emit radio waves no matter where it is. Therefore, the wireless transmission of data than the fixed transmission is more susceptible to theft or deception, causing problems: if a computer impersonates other computer's identity then the question arises as how to prevent the illegal data access. Secondly, the carrying of mobile computers is convenient and it is also easy for them to get stolen, therefore how can the data of the mobile computer theft be avoided to be received or transmitted. Moreover, in a mobile computing environment, the user freely makes use of arbitrary networks, then how can the flooding of mobile users be prevented resulting in the visited network environment of accidental or even malicious destruction. These are the security aspects of mobile database. In the mobile database, the se curity factor requires the effective management of mobile users. At present, generally the method adopted is: each system user has a unique symbol (ID), unified system of the ID management according to the security needs of access control. The main measures of safety technology are: first, on MC certification, prevent fraudulent access to non-registered $\mathrm{MC}$; second, encrypt the wireless path, in order to prevent third-party theft; third, provide identity protection to mobile users, preventing user location leaks or condition of being tracked.

\section{NEED FOR SMALL, EMBEDDED IN-MEMORY, MOBILE DBMS}

The minimum unit of the experimental setup is the broadcasting unit. As a unit of computation, the selection of the broadcasting unit has two advantages:

(1) the number of radio units than the absolute time can more objectively reflect the access request average response time, because in the experiment, environment is wired to simulate the wireless broadcast, so there is no phenomenon of loss of the broadcast data in the air, as the client responds to the access request and waits for the radio unit number is client processing power factors influence, is a more objective measurement units;

(2) the radio unit can more intuitively reflect in response to a request for a visit and waiting time, for example, broadcast cycle for 1600 broadcast unit broadcasting system, in a no local cache, the client response a request for access the average time is probably the entire cycle of half, namely 800 broadcast unit.

If the client has a local cache, and makes use of an algorithm to implement a management strategy, then access to the requested average response time is only 200 radio unit. Obviously, by comparison, the differences can clearly be reflected in local cache effects and various management strategies.

$$
S_{u+1}=S_{u^{\prime}}+P_{v} \cdot \frac{T}{2 X_{i}}=S_{u^{\prime}}+C \cdot \frac{P_{v}}{X_{v}}
$$

The rationality and feasibility of the experimental model is the key to the rationality of the experimental results. This model is divided into two key parts: server model and client model. The server mainly uses the broadcast scheduling algorithm to achieve data broadcast scheduling, and the client mainly generates continuous access requests and realizes local Cache management. The data page is of uniform size as a transmission unit, which does not affect the superiority of the algorithm and system performance analysis of the experimental results. Moreover it also reduces many external influences, reflecting more essential differences between the various algorithms. The experimental model tests the capability of the system of temporal logic unit known as the radio unit. A radio unit refers to the time spent by broadcasting a data page, and the radio unit of absolute time and transmission media and operating environment.

Because once the server broadcast program is established, the client can only passively listen from the server broadcast channel to performance and the system of any single client and other client is rendered irrelevant. However, with the increasing number of clients, server side data scheduler should correspondingly adjust according to the access requests. Therefore, the downlink of the broadcast channel determines the mutual independence and dependence of the different mobile clients.

The impact of the radio program can be reflected in a timely manner, as a client can be used to simulate the operation in a multiple client environment (Fig. 7). 


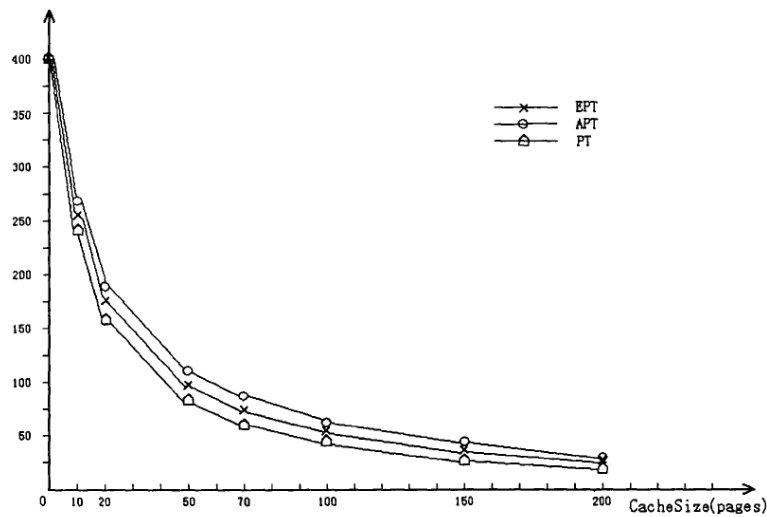

Fig. (7). Cache size curve.

\section{(Repeated) (Repeated)}

All the above synchronization processes are enabled using EAN.UCC XML communication standards (called Business Message Standards). Here, the major characteristics of the communication standards specified in various reports are summarized published by GS1 (the EAN.UCC System standards for the GDSN are available at www.gs1.org/GDS under the technical section).

The schemas are organized into three distinct layers: Envelope, Message, and Document. Every layer is wrapped inside the other and combines to form an XML message. The schemas are applied to make each layer independent of each other. A change in one layer does not affect other layers, achieving higher reuse. Several business documents can be packed and transmitted in one envelope, making transmission more efficient. The Envelope layer carries fundamental transport information such as sender, recipient, message identification, security, and guaranteed message delivery information. It allows using standards such as EDIINT-AS2, SOAP or ebXML although the currently recommended and supported standard is AS2 Envelope. The transport method used by AS2 standard is HyperText Transfer Protocol. The Message layer specifies actions as commands (e.g. "Add", "Delete"). The Message layer has three primary components: transaction, command, and an interface to the Document Layer. Document layer holds the actual business documents that are exchanged between trading partners by containing either the complete document itself, an internal reference to a document (GLN, GTIN), or an external reference to a document (URL). The documents are organized following typical business processes, such as order business process and delivery business process.

The first and most critical challenge is the need to achieve internal data synchronization as a pre-requisite for GDS. The meaning of internal data synchronization is two- fold: first, the organization needs to clean the data and have a single version of item and partner data on which its internal information systems can rely on. Secondly, it needs to establish an organization-wide, ongoing business process, and a set of rules to maintain the data in that manner [Intel Corporation, 2004]. The need for internal data synchronization is critical. Many organizations have not built corporate-wide data integrity, having several versions of item and pricing information at different sections/locations. Moreover, data are sometimes incorrectly keyed-in and the data validation process is not carried out in a timely fashion. All of these contribute to the problem of different versions of the same story. Furthermore, the organization needs to determine what information about the item should be maintained internally and shared with its business partners with respect to the EAN/UCC attribute standards and partner's information requirements.

\section{CONCLUSION}

The mobile database and its application are introduced in this paper. The characteristics of data synchronization technology are analyzed and a realization model is given based on the conventional remote data access and merged replication technologies. Taking mobile levy of maintenance costs of waterway as an example, the process of data synchronization is described in detail.

\section{CONFLICT OF INTEREST}

The authors confirm that this article content has no conflicts of interest.

\section{ACKNOWLEDGEMENTS}

Declared none.

\section{REFERENCES}

[1] S. Acharya, "Broadcast disks-dissemination-based data management for asymmetric communications environment", $\mathrm{PhD}$ Thesis, Brown University, Providence, RI, USA, 2008.

[2] S. Acharya, M. Franklin and S. Zdonik, "Dissemination-based data delivery using broadcast disks", IEEE Personal Communications, vol. 2 , no. 6 , pp. 50-60, 2005.

[3] S. Acharya, M. Franklin and S. Zdonik, "Prefetching from a broadcast disk", Proceedings of $12^{\text {th }}$ International Conference on Data Engineering, pp. 276-285, 2006.

[4] K. Stathatos, N. Roussopoulos, and J.S. Baras, "Adaptive data broadcast in hybrid networks," Proceedings of $23^{\text {rd }}$ International Conference on Very Large Data Bases, Morgan Kaufmann Publishers Inc: San Francisco, CA, USA, 1997.

[5] J.X. Yu, T. Sakata, and K. Tan, "Statistical estimation of access frequencies in data broadcasting environments", Wireless Networks, vol. 6, pp. 89-98, 2010. 